\title{
Markov-Perfect Nash Equilibria in Models With a Single Capital Stock
}

\author{
Engelbert J. Dockner* \\ Department of Finance and \\ Vienna Graduate School of Finance \\ University of Vienna, Brünner Straße 72, A-1210, Austria \\ engelbert.dockner@univie.ac.at \\ and \\ Florian O.O. Wagener \\ Center for Nonlinear Dynamics in Economics and Finance, CeNDEF \\ Universiteit van Amsterdam, Roetersstraat 11, 1018 WB Amsterdam, The Netherlands \\ f.o.o.wagener@uva.nl
}

15th May 2006

\begin{abstract}
Many economic problems can be formulated as dynamic games in which strategically interacting agents choose actions that determine the current and future levels of a single capital stock. We study necessary conditions that allow us to characterize Markov perfect Nash equilibria (MPNE) for these games. These conditions result in an auxiliary system of ordinary differential equations that helps us to explore stability, continuity and differentiability of MPNE. The techniques are used to derive detailed properties of MPNE for several games including the exploitation of a finite resource, the voluntary investment in a public capital stock, and the inter-temporal consumption of a reproductive asset.
\end{abstract}

JEL classification: C73; D92; Q22

Keywords: Capital accumulation games, Markov equilibria, Resource games, Differential games

* Corresponding author. 


\section{Introduction}

Many economic problems can be formulated as dynamic games in which strategically interacting agents choose actions that determine the current and future levels of a single capital stock. Consider, for example, a single stock of an exhaustible or reproductive resource that is simultaneously exploited by several agents that do not cooperate. Each agent chooses an extraction strategy to maximize the discounted stream of future utility. The actions taken by agents not only determine their levels of utility but also the level of the capital stock. Alternatively, look at the problem that agents voluntarily contribute to a single public stock of capital, like a park or a church. They choose their contributions (investments in the public stock of capital) to maximize the discounted stream of utility from consuming the public stock net of investment costs. Private investment builds up the public stock of capital that eventually can be consumed by all agents.

Both examples have several things in common. First, the actions taken by agents determine the size of a single capital stock that fully describes the current state of the economic system. Second, in case there is no mechanism that forces players to coordinate their actions, they will act strategically and play a noncooperative game. Third, the equilibrium outcome will critically depend on the strategy spaces available to the agents.

We make use of these features and formulate a differential game in which agents act non-cooperatively and use Markov strategies. We provide a detailed analysis of Markov perfect Nash equilibria (MNPE) for this class of differential games with a single capital stock and discuss several economic examples that belong to this class.

In a differential game, strategically interacting agents try to maximize an inter-temporal objective function, by choosing a strategy that results in an action at every point in time. ${ }^{1}$ Collectively, these actions influence the state of the economic system and how it evolves over time. The evolution of the system as a result of the agents' strategies is captured in the system dynamics.

There is a wide choice of possible strategies taken by the agents. They may choose a simple time profile of actions and precommit themselves to these fixed actions over the entire planning horizon. In that case players are using open-loop strategies. Alternatively players might choose Markov strategies. In this case they condition their actions on the current state of the system and react immediately every time the state variable changes. When agents use feedback or Markov strategies they are not required to precommit. Instead they play credible strategies if they are derive through backward induction.

To better understand the difference between open-loop and Markov strategies let us look at the following example of several agents strategically exploiting the same renewable resource. In case the fisheries use open-loop strategies they specify a time path of fishing effort at the beginning of the game and commit themselves to stick to these preannounced actions over the entire planning horizon. If alternatively they use Markov or feedback strategies they choose decision rules that determine current actions as a function of the current stock of the resource. Markov decision rules capture the strategic interactions present in a dynamic game. If the rival fishery makes a catch today that necessarily results in a lower level of the fish stock, the opponent reacts with an action that takes this change in the stock into account. In that sense Markov strategies capture all the features of strategic interactions.

Assuming that agents use Markov strategy spaces we restrict our attention to the derivation of subgame perfect Nash equilibria. These strategies have the property that a player knowing the strategies of the other

\footnotetext{
${ }^{1}$ For a general introduction to the theory of differential games we refer the reader to [9].
} 
players cannot gain by unilaterally deviating from his equilibrium strategy. Finding Markov Nash equilibrium strategies of differential games, even if the game is of the linear-quadratic type, is a formidable analytical problem. For instance, to find a MPNE in the general case of $n$ players and $m$ state variables leads to the problem of determining solutions of a system of $n$ coupled nonlinear implicit $m$-dimensional partial differential equations (PDE). Only in the case that the economic system can be described by a single state variable (a single capital stock) will the system of PDE's collapse to a system of ordinary differential equations that is much easier to deal with. Because of this, the article focusses on the least complex case $m=1$. As it turns out there are many economic problems that result in a dynamic game with a single capital stock.

Consider the case of $n$ agents non-cooperatively exploiting a single exhaustible or renewable resource. The resource stock is the single state variable and agents choose extraction strategies to maximize the present value of utility over a given planning horizon. Markov perfect equilibria for these classes of games have been studied, for example, by Levhari and Mirman [16], Sundaram [20], Benhabib and Radner [2], Clemhout and Wan [5], Dutta and Sundaram [12], Dockner and Sorger [8], Rincon-Zapatero et al. [19] and more recently Benchekroun [1].

As a second class of models consider the private investment in a public capital stock. The capital stock is the single state variable and agents choose investment strategies to maximize the present value of future net utility. Utility is derived from the available stock of public capital. This problem of private investment in a single public capital stock has been formulated by Fershtman and Nitzan [14] and MPNE been studied by Wirl [22], and in a discrete time framework by Marx and Matthews [18] and Dockner and Nishimura [11].

Dynamic games with a single capital stock can also be applied to study transboundary pollution control. The emissions of two or more countries accumulate a single stock of pollution. Countries derive utility from consumption but production results in emissions that accumulate and generate costs. Markov equilibria for these types of games have been studied by Dockner and Long [10] and Dockner et al. [7]. Finally, environmental economists have recently started to explore equilibria in the shallow lake problem. This problem is structurally similar to the exploitation of a single renewable resource stock but with a nonconcave production function. Recent papers dealing with the shallow lake problem include Brock and Starrett [4], Mäler et al. [15], and Wagener [21].

In this paper we formulate a class of differential games in which the actions of the agents influence a single capital stock, the state variable, and develop a solution methodology, whose core is formed by necessary conditions that have to be satisfied by Markov strategies. For the $n$-player case, an $n$-dimensional system of explicit ordinary non-autonomous differential equations is found, which has to be satisfied by Markov strategies at all points for which they are continuously differentiable. Moreover, necessary conditions are given for possible failures of differentiability and continuity of the Markov equilibrium strategies. Points where the Markov strategies are continuous but not differentiable can very conveniently be described by singularities of an autonomous auxiliary $(n+1)$-dimensional vector field, whose solution trajectories are the graphs of the equilibrium strategies. In the important special case that all players have the same utility functionals, it is useful to focus on a symmetric equilibrium in which all agents play the same strategy. The symmetric equilibrium is the solution of an ordinary non-autonomous differential equation. In this case, it is possible to draw the associated two-dimensional auxiliary vector field, which yields detailed information about the qualitative properties of the symmetric Markov equilibrium.

The approach of using an auxiliary system of differential equations to characterize Markov equilibria has also been used by Rincon-Zapatero et al. [19] and Xepapadeas and co-workers [17, 23]. Contrary to our approach, however, these authors derive sufficient conditions and do not deal with the problems of 
differentiability and continuity of equilibrium strategies. Being able to deal with non-differentiable and discontinuous equilibrium strategies in a systematic manner is very important and fills a gap in the literature of economic applications of differential games.

The article is organized as follows. In section 2, necessary conditions are derived which have to hold at points where a Markov strategy is differentiable, or continuous, or discontinuous, respectively. Section 3 illustrates these conditions by determining Markov equilibria for different applications of differential games with a single capital stock: (i) extraction of exhaustible resources; (ii) voluntary provision of public goods; (iii) management of an economical-ecological system, known as the shallow lake system; and (iv) exploitation of renewable resources. Section 4 concludes.

\section{Markov strategies}

In this section we formulate a differential game in which $n$-players choose Markov strategies, $u_{i}(x)$, to maximize an inter-temporal objective function. The strategies determine the level of a single capital stock, $x$, that is governed by the state dynamics. For this game we derive necessary conditions for a Markov perfect equilibrium.

Recall that such an equilibrium is by definition a vector-valued function $u: X \rightarrow \mathbb{R}^{n}$, such that if the strategies of all players except player $i$ are given by the component functions $u_{j}(x), j \neq i$, then $u_{i}(x)$ would be the optimal feedback control for player $i$ of the resulting optimal control problem.

2.1 Basic idea. The basic idea for obtaining the necessary conditions for a MPNE can be described as follows. Consider an $n$ player game, where each player has a payoff functional

$$
\partial_{i}\left[u_{i}\right]=\int_{0}^{\infty} L_{i}(x, u) \mathrm{e}^{-\rho t} \mathrm{~d} t .
$$

Here $x \in X$, where $X$ is an open subinterval of the real line $\mathbb{R}$, and $u$ denotes the vector $u=\left(u_{1}, \cdots, u_{n}\right) \in$ $\mathbb{R}^{n}$ of the strategies of the players. For known $u$, the state equation

$$
\dot{x}=f(x, u)
$$

determines the evolution of the system.

Given the strategies of the other players in feedback form, the Pontryagin necessary conditions yield an autonomous system of differential equations for the best response strategy of player $i$; let this system be solved by functions $\left(x(t), u_{i}(t)\right)$. Since the state space is one-dimensional, the map $t \mapsto x(t)$ can be seen as a variable transformation locally at some value $t=t_{0}$ if $\dot{x}\left(t_{0}\right) \neq 0$. Using the inverse $t=t(x)$ of this transformation, we obtain from the pair $(x(t), u(t))$ a strategy in feedback (Markov) form: $u_{i}(x)=u_{i}(t(x))$.

Using this basic idea, an explicit system of ordinary differential equations will be derived for the $u_{i}(x)$, which is necessarily satisfied by Markov strategies at points where they are simultaneously continuously differentiable.

This condition leads naturally to the question of what can be said about points where the continuous differentiability fails to hold: such points are investigated in subsections 2.4 and 2.6, where the respective possibilities of discontinuities in the first derivative of the control function, and in the control itself are investigated. 
2.2 The associated control problem. For ease of notation, it is assumed that the strategies of players 1 to $n-1$ are fixed, leaving player $n$ to solve for his optimal response. Let $\hat{u}=\hat{u}(x)=\left(u_{1}(x), \cdots, u_{n-1}(x)\right)$ denote this vector of fixed strategies. Player $n$ optimizes

$$
\partial_{n}\left[u_{n}\right]=\int_{0}^{\infty} L_{n}\left(x, \hat{u}(x), u_{n}\right) \mathrm{e}^{-\rho_{n} t} \mathrm{~d} t,
$$

under the constraint that

$$
\dot{x}=f\left(x, \hat{u}(x), u_{n}\right), \quad x(0)=x_{0} .
$$

The functions $L_{j}, j=1, \cdots, n$ and $f$ are assumed to be sufficiently many times continuously differentiable. Introduce the Pontryagin function ${ }^{2} \mathcal{P}_{n}$ of player $n$ 's optimal control problem

$$
\mathcal{P}_{n}\left(x, p_{n}, \hat{u}, u_{n}\right)=-L_{n}\left(x, \hat{u}, u_{n}\right)+p_{n} f\left(x, \hat{u}, u_{n}\right),
$$

with $p_{n}$ as the costate variable. Pontryagin's minimal principle requires that $\mathcal{P}_{n}\left(x, p_{n}, U\right)$ takes a minimum for $U=u_{n}$. If it is assumed that for given $x$ the function $\hat{u}(x)$ is continuously differentiable, and that the minimum is interior, then necessarily

$$
\frac{\partial \mathcal{P}_{n}}{\partial u_{n}}=0 .
$$

Note that from this equation, the co-state $p_{n}$ can be solved as a function $p_{n}=q_{n}\left(x, \hat{u}, u_{n}\right)$. Inversely, if the condition is imposed that $\mathcal{P}_{n}$ is (locally) strictly convex in $u_{n}$ for all $x, \hat{u}$ and $p_{n}$, then the control $u_{n}$ can be solved smoothly from equation (3) as a function $u_{n}=v_{n}\left(x, \hat{u}, p_{n}\right)$.

More generally, we may consider the case that the control variable $u_{n}$ is constrained to some set $S$. As this case is not our main concern, we shall comment on it briefly and we will not try to achieve utmost generality of our results in this direction. In particular, we shall assume that the set $S$ is given as

$$
S=S_{x, \hat{u}}=\left\{u_{n} \mid h_{n}(x, \hat{u}, u) \leq 0\right\},
$$

and that on the boundary of $S$ (i.e. for $h_{n}=0$ ) the derivative $\partial h_{n} / \partial u_{n}$ does not vanish. The necessary conditions read in this case as

$$
\frac{\partial \mathcal{P}_{n}}{\partial u_{n}}=\mu_{n} \frac{\partial h_{n}}{\partial u_{n}}, \quad \mu_{n} h_{n}=0, \quad \mu_{n} \geq 0, \quad h_{n} \leq 0 .
$$

Imposing strict convexity of $\mathcal{P}_{n}$ and quasi-convexity of $h_{n}$ with respect to $u_{n}$, we can again solve uniquely the control $u_{n}$ from the necessary conditions, together with the multiplier $\mu_{n}$, but now only as a continuous function $v_{n}\left(x, \hat{u}, p_{n}\right)$. However, the discontinuities in the derivatives of $v_{n}$ occurr only for those values of $p_{n}$ for which both $h_{n}=0$ and $\mu_{n}=0$.

Introduce the (minimized) Hamiltonian

$$
H_{n}\left(x, p_{n}\right)=\mathcal{P}_{n}\left(x, p_{n}, \hat{u}(x), v_{n}\left(x, \hat{u}(x), p_{n}\right)\right) ;
$$

then an optimal response of player $n$ has to satisfy the following equations for $x(t)$ and $p_{n}(t)$ :

$$
\dot{x}=\frac{\partial H_{n}}{\partial p_{n}}, \quad \dot{p}_{n}=\rho_{n} p_{n}-\frac{\partial H_{n}}{\partial x} .
$$

\footnotetext{
${ }^{2}$ This function is usually called the Hamilton function in the optimal control literature; however, we like to reserve that name for the function $H_{n}$ introduced below, also called the 'minimized Hamilton function', which depends only on states and co-states.
} 
See [3] for more details on Pontryagin necessary conditions.

For an optimal solution $\left(x(t), u_{n}(t)\right)$ beginning at a state $x_{0}$, the value (or valuation) function of player $n$ is given by

$$
V_{n}\left(x_{0}\right)=\int_{0}^{\infty} L_{n}\left(x(t), \hat{u}(x(t)), u_{n}(t)\right) \mathrm{e}^{-\rho_{n} t} \mathrm{~d} t .
$$

It satisfies the equation of Hamilton-Jacobi-Bellman:

$$
\rho_{n} V_{n}(x)=-H_{n}\left(x, \frac{\mathrm{d} V_{n}}{\mathrm{~d} x}(x)\right) .
$$

As $\frac{\mathrm{d} V_{n}}{\mathrm{~d} x}(x(t))=p_{n}(t)$, this could also be written as $\rho_{n} V_{n}=-H_{n}\left(x, p_{n}\right)$.

2.3 Derivation of necessary conditions. From the preceding subsection it is clear that each individual player has to solve an optimal control problem given the Markov strategies of the rivals. Hence, we can make use of the system (4) to derive necessary conditions for a stationary Markov equilibrium.

THEOREM 1. Let $u(x)=\left(u_{1}(x), \cdots, u_{n}(x)\right)$ be Markov Nash equilibrium strategies of the differential game with payoffs (1) and state equation (2). Moreover, let $v_{i}$ satisfy equation (3) with $n$ replaced by $i$.

If $u(x)$ is continuously differentiable in a neighborhood of a point $x_{0}=x\left(t_{0}\right)$ such that $f\left(x_{0}, u\left(x_{0}\right)\right) \neq 0$, then necessarily the differential equations

$$
\frac{\mathrm{d} u_{i}}{\mathrm{~d} x} f+\sum_{j \neq i}\left(\frac{\partial v_{i}}{\partial p_{i}} \frac{\partial \mathcal{P}_{i}}{\partial u_{j}}-\frac{\partial v_{i}}{\partial u_{j}} f\right) \frac{\mathrm{d} u_{j}}{\mathrm{~d} x}=\frac{\partial v_{i}}{\partial x} f+\frac{\partial v_{i}}{\partial p_{i}}\left(\rho_{i} p_{i}-\frac{\partial \mathcal{P}_{i}}{\partial x}\right) .
$$

evaluated at $p_{i}=q_{i}\left(x, u_{1}, \cdots, u_{n}\right)$ for all $i$, are satisfied at $t_{0}$, for every $i \in\{1, \ldots, n\}$.

\section{Proof}

Writing the equation for $\dot{p}_{n}$ in full (see equation (4)) and using equation (3) to simplify yields

$$
\dot{p}_{n}=\rho_{n} p_{n}+\frac{\partial L_{n}}{\partial x}+\frac{\partial L_{n}}{\partial \hat{u}} \frac{\mathrm{d} \hat{u}}{\mathrm{~d} x}-p_{n} \frac{\partial f}{\partial x}-p_{n} \frac{\partial f}{\partial \hat{u}} \frac{\mathrm{d} \hat{u}}{\mathrm{~d} x} .
$$

Note that expressions like $\partial L_{n} / \partial \hat{u} \cdot \mathrm{d} \hat{u} / \mathrm{d} x$ are shorthand for $\sum_{j=1}^{n-1} \partial L_{n} / \partial \hat{u}_{j} \cdot \mathrm{d} \hat{u}_{j} / \mathrm{d} x$.

As mentioned in the introduction to this section, if $x^{\prime}(t) \neq 0$, then $x(t)$ can be seen as a local coordinate transformation. In order not to burden notation too much, both $\hat{u}(x)$ and $\hat{u}(x(t))$ shall be denoted by $\hat{u}$; the meaning should always be clear from the context. The chain rule yields

$$
\frac{\mathrm{d} \hat{u}}{\mathrm{~d} x}(x(t))=\frac{1}{\dot{x}(t)} \frac{\mathrm{d}}{\mathrm{d} t} \hat{u}(x(t))=\frac{1}{f} \frac{\mathrm{d}}{\mathrm{d} t} \hat{u}(x(t)) .
$$

This implies for the $\dot{p}_{n}$-equation that

$$
\dot{p}_{n}=\rho_{n} p_{n}+\frac{\partial L_{n}}{\partial x}-p_{n} \frac{\partial f}{\partial x}+\frac{1}{f}\left(\frac{\partial L_{n}}{\partial \hat{u}}-p_{n} \frac{\partial f}{\partial \hat{u}}\right) \frac{\mathrm{d} \hat{u}}{\mathrm{~d} t} .
$$

Introducing $u_{n}(t)$ by setting $u_{n}(t)=v_{n}\left(x(t), \hat{u}(x(t)), p_{n}(t)\right)$, it follows that

$$
\dot{u}_{n}=\left(\frac{\partial v_{n}}{\partial x}+\frac{\partial v_{n}}{\partial \hat{u}} \frac{\mathrm{d} \hat{u}}{\mathrm{~d} x}\right) \dot{x}+\frac{\partial v_{n}}{\partial p_{n}} \dot{p}_{n} .
$$


Substituting $f$ for $\dot{x}$ and the right hand side of (6) for $\dot{p}_{n}$ yields

$$
\begin{aligned}
\dot{u}_{n} & =\left(\frac{\partial v_{n}}{\partial x}+\frac{\partial v_{n}}{\partial \hat{u}} \frac{\mathrm{d} \hat{u}}{\mathrm{~d} x}\right) f+\frac{\partial v_{n}}{\partial p_{n}}\left(\rho_{n} p_{n}-\frac{\partial H_{n}}{\partial x}\right) \\
& =\frac{\partial v_{n}}{\partial x} f+\frac{\partial v_{n}}{\partial p_{n}}\left(\rho_{n} p_{n}-\frac{\partial \mathcal{P}_{n}}{\partial x}\right)-\left(\frac{\partial v_{n}}{\partial p_{n}} \frac{\partial \mathcal{P}_{n}}{\partial \hat{u}}-\frac{\partial v_{n}}{\partial \hat{u}} f\right) \frac{\mathrm{d} \hat{u}}{\mathrm{~d} x} .
\end{aligned}
$$

Using that $u_{j}(t)=\hat{u}_{j}(x(t))$ satisfies $\dot{u}_{j}=f \mathrm{~d} \hat{u}_{j} / \mathrm{d} x$, and bringing all derivatives $\dot{u}_{j}$ to one side yields

$$
\dot{u}_{n}+\sum_{j=1}^{n-1}\left(\frac{1}{f} \frac{\partial v_{n}}{\partial p_{n}} \frac{\partial \mathcal{P}_{n}}{\partial u_{j}}-\frac{\partial v_{n}}{\partial u_{j}}\right) \dot{u}_{j}=\frac{\partial v_{n}}{\partial x} f+\frac{\partial v_{n}}{\partial p_{n}}\left(\rho_{n} p_{n}-\frac{\partial \mathcal{P}_{n}}{\partial x}\right) .
$$

Of course, the label $n$ is by no means special; hence for every $u_{j}$ an equation of the form (7) can be obtained. Put together, a system of equations linear in the $\dot{u}_{j}$ is obtained, which can be solved (under appropriate determinant conditions) to yield a system of (nonlinear) ordinary differential equations for the $u_{j}$. Changing to $\mathrm{d} u_{n} / \mathrm{d} x$ using the chain rule yields the result.

2.4 Corner points. Equation (5) also answers the question of when a continuous equilibrium Markov strategy $u(x)$ may fail to be differentiable at certain (isolated) points.

Introduce the square matrix $A(x)=\left(a_{i j}(x, u)\right)_{i j=1}^{n}$ with elements $a_{i i}=f(x, u)$ and $a_{i j}(x, u)=\partial v_{i} / \partial p_{i}$. $\partial \mathcal{P}_{i} / \partial u_{j}$ if $i \neq j$ as well as the vector $b(x)=\frac{\partial v_{i}}{\partial x} f+\frac{\partial v_{i}}{\partial p_{i}}\left(\rho_{i} p_{i}-\frac{\partial \mathcal{P}_{i}}{\partial x}\right)$. Based on these we are able to show our next result.

THEOREM 2. Assume that $u=u(x)$ is a Markov Nash equilibrium strategy of the differential game with payoffs (1) and state equation (2), that $u$ is continuous in a neighborhood $U$ of $x_{0}$ and continuously differentiable in $U \backslash\left\{x_{0}\right\}$, and that $A\left(x_{0}, u\left(x_{0}\right)\right)$ is invertible. Then $u$ is differentiable at $x_{0}$.

\section{Proof}

Rewrite equation (5) in the following schematic form (recall that $u=u(x) \in \mathbb{R}^{n}$ ):

$$
A(x, u(x)) \frac{\mathrm{d} u}{\mathrm{~d} x}(x)=b(x, u(x)) .
$$

If $A(x, u(x))$ is invertible, this can be rewritten as

$$
\frac{\mathrm{d} u}{\mathrm{~d} x}(x)=A(x, u(x))^{-1} b(x, u(x))=F(x) .
$$

Read differently, if the vector field $F(x)$ exists it gives for every $x$ the only possible value for $\mathrm{d} u / \mathrm{d} x$.

Hence, let $x_{0}$ be a point such that the left and right limits of $\mathrm{d} u / \mathrm{d} x$ exist as $x$ tends to $x_{0}$, then there are the following two possibilities. Either $F\left(x_{0}\right)$ exists, it is the limit of $\mathrm{d} u / \mathrm{d} x$, and $u$ is continuously differentiable at $x_{0}$. Or $F\left(x_{0}\right)$ fails to exist; but this can happen only if $A(x)$ fails to be invertible at $x_{0}$.

2.5 Auxiliary system. Another way to understand the occurrence of corner points is to consider what will be called the auxiliary system to the system given by equation (8).

Recall the definition of the adjoint matrix $A^{*}$ of a given matrix $A$ : it is the matrix whose elements are the cofactors of $A$, which are obtained by deleting the $i$ 'th row and $j$ 'th column of $A$ and taking the determinant 
of the remaining matrix. We have that $A A^{*}=(\operatorname{det} A) I$, where $I$ is the identity matrix; hence $A^{-1}=$ $(\operatorname{det} A)^{-1} A^{*}$. Note that equation (8) implies

$$
\frac{\mathrm{d} u}{\mathrm{~d} x}=A^{-1} b=\frac{1}{\operatorname{det} A} A^{*} b .
$$

Now consider the system of differential equations

$$
\left\{\begin{array}{l}
\tilde{u}^{\prime}=A^{*}(\tilde{x}, \tilde{u}) b(\tilde{x}, \tilde{u}) \\
\tilde{x}^{\prime}=\operatorname{det} A(\tilde{x}, \tilde{u})
\end{array}\right.
$$

here $\tilde{u}^{\prime}$ is differentiation with respect to some real parameter $s \cdot{ }^{3}$ By the chain rule, it follows that whenever $\tilde{x}^{\prime} \neq 0$, then $\frac{\mathrm{d} u}{\mathrm{~d} x}(\tilde{x}(s))=\tilde{u}^{\prime}(s) / \tilde{x}^{\prime}(s)$. Hence if $(\tilde{x}(s), \tilde{u}(s))$ are solution curves of the auxiliary system, and if for $s=s_{0}$ we have that $\tilde{x}^{\prime}\left(s_{0}\right) \neq 0$, then the equation $\tilde{x}(s)=x$ can be solved for $s=s(x)$, and in a neighborhood of $x\left(s_{0}\right)$ the function $\tilde{u}(s(x))$ yields a solution of (8). The system given by equation (10) is an auxiliary system to equation (9).

More generally, a system $u^{\prime}=k(x, u), x^{\prime}=h(x, u)$, with $k(x, u) \in \mathbb{R}^{n}$ and $h(x, u) \in \mathbb{R}$, is an auxiliary system to (9), if

$$
\frac{1}{h} k=A^{-1} b
$$

2.6 Jump points Note that theorem 1 yields a necessary condition at points where the equilibrium strategies are continuously differentiable. Here, we look for necessary conditions that have to hold if the equilibrium strategy has an isolated jump discontinuity.

Let $p(x)=\left(p_{1}(x), \cdots, p_{n}(x)\right)=\left(q_{1}(x, u(x)), \cdots, q_{n}(x, u(x))\right)$, with $q_{j}(x, u)$ as introduced in subsection 2.2. The result can then be stated as follows.

THEOREM 3. Assume that the value function $V_{i}$ of player $i$ is continuous. It is necessary that

$$
\lim _{x \uparrow x_{*}} H_{j}(x, p(x))=\lim _{x \downarrow x_{*}} H_{j}(x, p(x)),
$$

for every $x_{*} \in X$ and all $j=1, \cdots, n$.

\section{Proof}

In the appendix we state the conditions that imply the continuity of the value functions $V_{i}$. Recall that the value function of player $i$ satisfies $\rho V_{i}(x)=-H_{i}\left(x, p_{i}(x)\right)$. Given $c=-\rho V_{i}(x)$, the only values possible for $p_{i}$ are those for which

$$
H_{i}\left(x, p_{i}\right)=c .
$$

The theorem follows from this.

\footnotetext{
${ }^{3}$ The relationship between the time derivative $\dot{x}=\frac{d x}{d t}$ and the derivative with respct to $s, x^{\prime}=\frac{d x}{d s}$ follows from$$
x^{\prime}=\frac{d x}{d s}=\frac{d x}{d t} \frac{d t}{d s}=f \frac{d t}{d s} .
$$ 


\section{REMARKS.}

1. The necessary condition stated in the Theorem cannot be used to identify the location of jump discontinuities but imposes a restriction on the jump size.

2. If $H_{n}$ is convex in $p_{n}$, then there are at most two solutions to the equation $H_{n}\left(x, p_{n}\right)=c$.

3. Let $u(x)$ be defined for all $x<x_{*}$, and assume that there is only one possibility for a nonzero jump at $x_{*}$, to a continuous strategy $\tilde{u}(x)$ defined for all $x \geq x_{*}$. Let

$$
\lim _{x \uparrow x_{*}} f(x, u(x))=A, \quad \text { and } \quad \lim _{x \downarrow x_{*}} f(x, \tilde{u}(x))=f\left(x_{*}, \tilde{u}\left(x_{*}\right)\right)=B .
$$

If the time evolution $x(t)$ is continuous and piecewise differentiable, then it is not possible that simultaneously $A>0$ and $B<0$. For, suppose it were the case. Then if $x\left(t_{0}\right)=x_{*}$, necessarily $x(t)=x_{*}$ for all $t>t_{0}$. Hence $B$ should be equal to 0 , contradicting the assumption.

\section{Applications}

The class of differential games introduced in the preceding section is fairly general and allows us to study Markov equilibria for a variety of different examples. Here we apply the techniques of the auxiliary system to four alternative models that have been dealt with in the literature: (i) the exploitation of an exhaustible resource (see [13], [19], and [5]), (ii) the voluntary provision of a public capital good (see [14] and [22]), (iii) the shallow lake problem (see [15], [21], and [4]) and (iv) the exploitation of a reproductive asset (see [2], [8], and [1]).

3.1 Exploitation of exhaustible assets. Let $x$ be the stock of some exhaustible resource, and let $u_{i}$ be player $i$ 's rate of exploitation of this resource. Assume that the objective function of this player is given by

$$
\mathcal{J}_{i}=\int_{0}^{\infty} L_{i}\left(u_{i}\right) \mathrm{e}^{-\rho t} \mathrm{~d} t
$$

where $L_{i}\left(u_{i}\right)$ is the instantaneous utility that exhibits positive and decreasing marginal utility. The stock dynamics of the resource is described by

$$
\dot{x}=-\sum_{i=1}^{n} u_{i}
$$

The function $\mathcal{P}_{i}$ is given by

$$
\mathcal{P}_{i}=-L_{i}\left(u_{i}\right)-p_{i} \sum_{i=1}^{n} u_{i},
$$

yielding $p_{i}=-L_{i}^{\prime}\left(u_{i}\right) \equiv-\frac{\mathrm{d} L_{i}\left(u_{i}\right)}{\mathrm{d} u_{i}}$ if $u_{i}>0$, and $p_{i}=0$ if $u_{i}=0$. Restricting the analysis on symmetric equilibria $u_{i}=u$, equation (5) becomes

$$
(n+(1-n) E(u)) u \frac{\mathrm{d} u}{\mathrm{~d} x}=\rho E(u) u,
$$


where $E(u)$ measures the inverse of relative risk aversion, i.e.,

$$
E(u)=-\frac{L_{i}^{\prime}(u)}{L_{i}^{\prime \prime}(u) u} .
$$

Based on equation (11) we can now characterize symmetric Markov equilibria for different types of utility functions (see [19]). In particular we distinguish two different cases. One in which preferences exhibit constant relative risk aversion (CRRA) and one in which consumers have preferences with constant absolute risk aversion (CARA).

Let us consider the case of constant relative risk aversion first. Here the utility functions of the agents are given by

$$
L_{i}\left(u_{i}\right)=\frac{u_{i}^{1-\eta}}{1-\eta}
$$

and the inverse of relative risk aversion is given by

$$
E(u)=\frac{1}{\eta}
$$

Equation (11) becomes

$$
\left(\frac{n-1}{\eta}-n\right) u \frac{\mathrm{d} u}{\mathrm{~d} x}=-\frac{\rho}{\eta} u
$$

which is solved by either $u(x)=0$, or, if $n>1 /(1-\eta)$, by the linear function

$$
u(x)=G x, \quad \text { where } G=\frac{\rho}{(1-\eta) n-1} .
$$

This is the symmetric Nash equilibrium strategy for all players; note that in equilibrium, the rate of extraction $G$ is proportional to the discount rate, and it decreases with the number of players. In the limiting case when agents have logarithmic utility, $L_{i}\left(u_{i}\right)=\ln u_{i}$ and the elasticity becomes $E(u)=1$, extraction rates are independent of the number of firms exploiting the resource. They are given by $u(x)=\rho x$.

In case of constant absolute risk aversion, consumer preferences are given by

$$
L_{i}\left(u_{i}\right)=-e^{-\alpha u}
$$

where $\alpha>0$ is the constant absolute risk aversion. In this case we get

$$
E(u)=\frac{1}{\alpha u}
$$

and equation (11) becomes

$$
\left(n+(1-n) \frac{1}{\alpha u}\right) u \frac{\mathrm{d} u}{\mathrm{~d} x}=\frac{\rho}{\alpha} .
$$

Under the assumptions that $u(0)=0$ this equation can be integrated to yield equilibrium extraction rates equal to

$$
u(x)=\frac{(n-1)+\sqrt{(1-n)^{2}+2 \rho n \alpha x}}{n \alpha} .
$$

These equilibrium strategies are decreasing both in the number of firms exploiting the resource and in the level of absolute risk aversion. 
3.2 Voluntary provision of a public good. Next we turn to the analysis of private investment in a public capital stock. This game was first looked at by Fershtman and Nitzan [14]. They assume that each agent derives quadratic utility form the consumption of the public capital stock and in case he invests in the stock faces quadratic costs. Fershtman and Nitzan solve both the open-loop game and the game with Markov strategies and find that the dynamic free rider problem is more severe when agents use linear Markov strategies. Wirl [22] challenges this results and studies the identical linear quadratic game but solves for non-linear Markov equilibria. He finds that if the discount rate is small enough non-linear Markov strategies can support equilibrium outcomes that are close to the efficient provision of the public capital. Here we use this example and demonstrate how our methodology can be used to replicate this results and extend them to include discontinuous and non-differentiable strategies.

The game. Each player $i$ voluntarily invests in the public capital stock at the rate $u_{i}$. The single public capital stock evolves according to

$$
\dot{x}=\sum_{j=1}^{n} u_{j}-\sigma x ;
$$

here $\sigma>0$ is the constant depreciation rate. Following Fershtman and Nitzan we assume that player $i$ 's utility functional is given by

$$
\partial_{i}=\int_{0}^{\infty}\left(a x-\frac{b}{2} x^{2}-\frac{1}{2} u_{i}^{2}\right) \mathrm{e}^{-\rho t} \mathrm{~d} t
$$

where $a, b>0$ are positive parameters. Note that compared to the formulation of [22], one parameter has been scaled away. The corresponding present value Hamiltonian becomes

$$
\mathcal{P}_{i}=\frac{1}{2} u_{i}^{2}-a x+\frac{b}{2} x^{2}+p_{i}\left(\sum_{j=1}^{n} u_{j}-\sigma x\right) .
$$

The function $v_{i}$ is found by minimizing $\mathcal{P}_{i}$ with respect to $u_{i}$ under the condition $u_{i} \geq 0$. This yields that

$$
v_{i}\left(p_{i}\right)=\left\{\begin{aligned}
-p_{i} & p_{i} \leq 0 \\
0 & p_{i}>0
\end{aligned}\right.
$$

In the symmetric case $u_{i}=u$, the system (5) reads as

$$
\frac{\mathrm{d} u}{\mathrm{~d} x}=\left\{\begin{array}{cl}
\frac{(\rho+\sigma) u-a+b x}{(2 n-1) u-\sigma x} & u(x)>0 \\
0 & u(x)=0
\end{array}\right.
$$

The corresponding auxiliary system is (in the region $u>0$ )

$$
\begin{aligned}
& x^{\prime}=(2 n-1) u-\sigma x, \\
& u^{\prime}=(\rho+\sigma) u-a+b x .
\end{aligned}
$$

Some phase curves of the auxiliary system are shown in figure 1. In this figure solutions of the auxiliary system are represented by drawn curves. They can, at least locally, be interpreted as the graphs of possible symmetric feedback strategies $u(x)$. Three curves are highlighted: the strategy $u(x)=0$ at the lower boundary of the control region, which is a special solution. The other two are examples of a non-differentiable and a non-continuous Markov strategy, respectively. The figure will be discussed in geometric terms. 


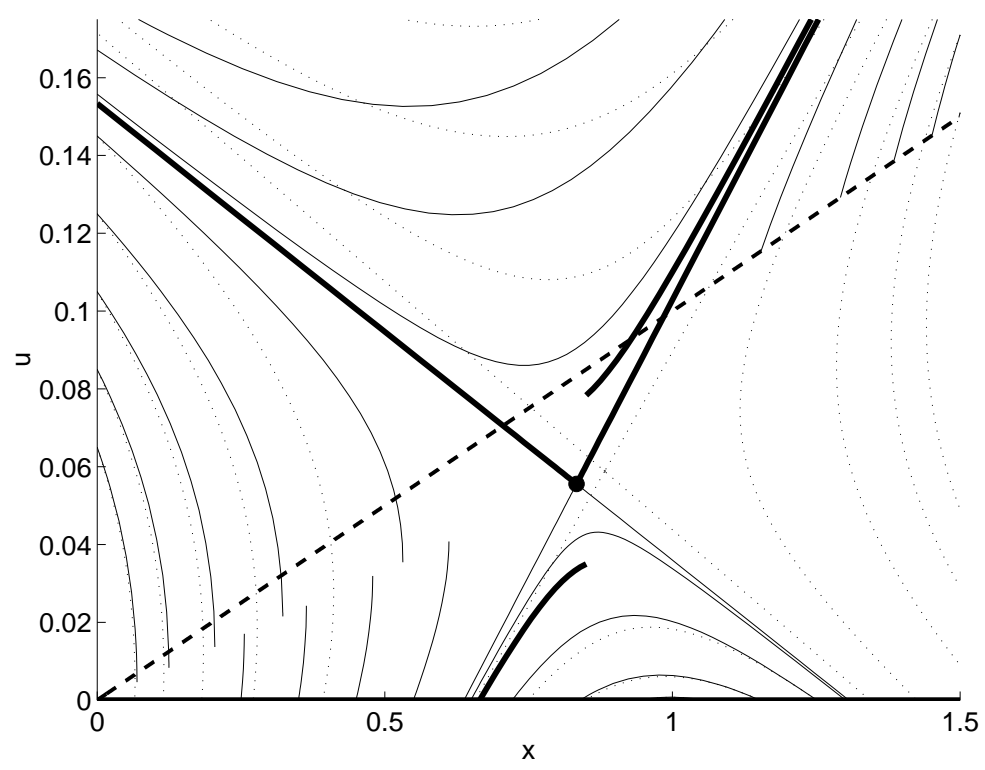

Figure 1: Solutions of the auxiliary system (drawn) and level curves of the value function (dotted) in the symmetric two player case of the voluntary provision of public goods game.

Stability of equilibria. First consider the line $\ell_{1}: n u-\sigma x=0$ of equilibria of the state equation (the broken line in the figure): the quantity $\dot{x}$ is positive above $\ell_{1}$, and negative below. Let $u(x)$ be a feedback strategy, and let $\left(x_{*}, u_{*}\right) \in \ell_{1}$ be an equilibrium such that $u_{*}=u\left(x_{*}\right)$. This equilibrium is stable if

$$
\frac{\mathrm{d}}{\mathrm{d} x} f\left(x_{*}, u\left(x_{*}\right)\right)<0 .
$$

This stability condition holds when

$$
\frac{\mathrm{d} u\left(x_{*}\right)}{\mathrm{d} x}<\frac{\sigma}{n}
$$

is satisfied. From the figure, it is readily apparent that points on $\ell_{1}$ close to the origin (lower left hand corner) are stable, while points on $\ell_{1}$ in the upper right hand corner are unstable. Hence there is a point on $\ell_{1}$ where equilibria change from stable to unstable; it is the unique point where a solution curve of the auxilary system touches the line $\ell_{1}$.

Forbidden region. Note the region in the lower right hand corner. Any solution of the auxiliary system ends (under the dynamics of the state equation) on the line $\ell_{2}:(2 n-1) u-\sigma x=0$ (not drawn in the picture: it is the line through the origin and the only steady state equilibrium $P=\left(x_{P}, u_{P}\right)$ of the auxiliary system). As this is coincidentally also the curve of maxima of the value function for given $x$, the strategies cannot 'jump away' from $\ell_{2}$. Neither can they stay there, for none of the points on $\ell$, excepting the origin, is an equilibrium of the state equation. Finally, continuation along solutions of the auxiliary system is impossible as well, for as the trajectories bend back, they cannot be represented by functions of the state variable any more.

Character of the strategies. This reasoning has to be modified slightly for the region in the lower left hand corner: here points that start at the line $\ell_{2}$ move away from it under state dynamics. Here solutions of the auxiliary system represent feedback strategies; these strategies are however not defined for all states. 
The feedback strategy which is formed by the upper two invariant manifolds of $P$ (two thickly drawn halflines in the figure) is of the type mentioned in subsection 2.4. Note that the corner point is on the line $\ell_{2}$, as predicted. The corners of the strategies ending (or beginning) on the line $u=0$ are of a different kind, since on that line the function $v(p)$ is non-differentiable.

The upper non-differentiable feedback strategy, as well as all continuous strategies above it are globally defined. The same holds for those in the region bounded by the lower two invariant manifolds of $P$, if we continue them by setting $u(x)=0$ after they hit the $x$-axis.

Jump points. Note the discontinuous strategy that 'jumps' over $P$ (also thickly drawn in the figure). At the jumping discontinuity, the players are indifferent between increasing or decreasing dynamics. This is similar to a Skiba point in open loop dynamics (see $[4,6,15,21]$ ).

3.3 Shallow lake. Consider the following environmental problem. There are $n$ farmers each applying fertilizer to his land. The amount of fertilizer used has two opposing effects. The more fertilizer a farmer uses, the higher is his harvest and hence revenue from farming. On the other hand the amount of fertilizer used eventually accumulates a stock of phosphorus in a shallow lake. The higher the phosphor level the higher are costs (for fresh water, less tourists come to his camping) to the farmer. Since the level of the phosphor stock is the result of activities of all farmers around the lake, the resulting problem can best be described by a differential game. The shallow lake system has been investigated in detail by $[6,15,21]$; we refer to these papers for background information.

Let the stock variable $x$ represent the amount of phosphorus in a shallow lake and let $u_{i}$ be the amount of fertilizer used by farmer $i$. Assuming a concave technology to produce farming output and quadratic costs coming from the stock $x$, player $i$ maximizes intertemporal utility

$$
\partial_{i}=\int_{0}^{\infty}\left(\log u_{i}-c_{i} x^{2}\right) \mathrm{e}^{-\rho t} \mathrm{~d} t .
$$

The level of phosphorus is assumed to evolve according to the following state equation:

$$
\dot{x}=f(x, u)=\sum_{i=1}^{n} u_{i}-b x+\frac{x^{2}}{x^{2}+1} ;
$$

where we have a constant rate of self-purification (sedimentation, outflow) and the nonlinear term $x^{2} /\left(x^{2}+\right.$ 1 ) is the result of biological effects in the lake.

For this differential game the function $\mathcal{P}_{i}$ is given by

$$
\mathcal{P}_{i}=-\left(\log u_{i}-c_{i} x^{2}\right)+p_{i}\left(\sum_{j=1}^{n} u_{j}-b x+\frac{x^{2}}{x^{2}+1}\right) .
$$

Hence, system (5) becomes

$$
f \frac{\mathrm{d} u_{i}}{\mathrm{~d} x}-u_{i} \sum_{j \neq i} \frac{\mathrm{d} u_{j}}{\mathrm{~d} x}=-(\rho+b) u_{i}+2 c_{i} u_{i}^{2} x+\frac{2 u_{i} x}{\left(x^{2}+1\right)^{2}} .
$$




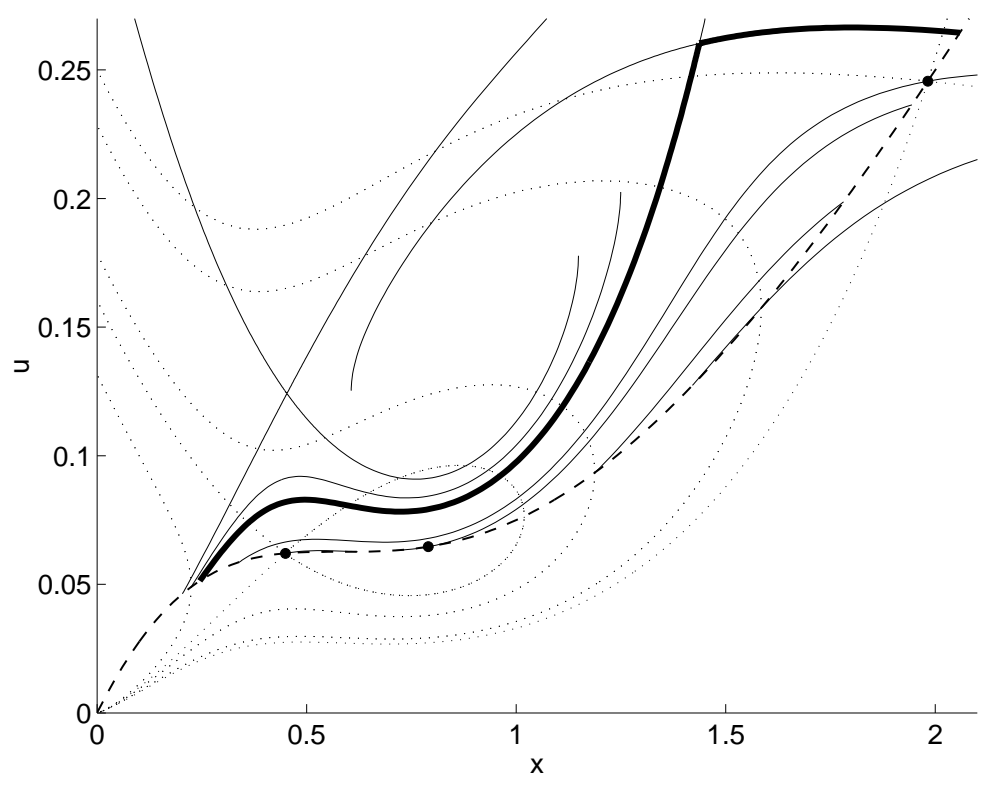

Figure 2: Solutions of the auxiliary system (solid) and level curves of the value function (dotted) in the symmetric two player case of the shallow lake game. The highlighted curve is an example of a non-differentiable feedback strategy.

If we restrict our attention to symmetric objective functionals $c_{i}=c$ it is possible to derive symmetric equilibrium strategies $u_{i}=u$. In this case system (5) reduces to

$$
\frac{\mathrm{d} u}{\mathrm{~d} x}=\frac{1}{f-(n-1) u}\left(-(\rho+b) u+2 c u^{2} x+\frac{2 u x}{\left(x^{2}+1\right)^{2}}\right) .
$$

Based on this the auxiliary system is given by

$$
\left(\begin{array}{l}
x^{\prime} \\
u^{\prime}
\end{array}\right)=\left(\begin{array}{l}
u-b x+\frac{x^{2}}{x^{2}+1} \\
-(\rho+b) u+2 c u^{2} x+\frac{2 u x}{\left(x^{2}+1\right)^{2}}
\end{array}\right) .
$$

Solutions to the auxiliary system are given in figure 2. It is shown that the symmetric equilibrium includes non-smooth strategies as well as multiple steady states.

Finally notice that the auxiliary system does not depend on the number of agents, and therefore coincides with the state-control system of the shallow lake optimal control problem. The optimal control problem is analyzed in detail in [21].

3.4 Exploitation of reproductive assets. As the final example consider the problem where $n$ agents strategically exploit a single reproductive asset, like fish or other species (see [8]). The reproduction of the stock $x$ occurs at rate $h(x)$, whereas player $i$ extracts the stock at rate $u_{i}$. Hence, the state dynamics is given by

$$
\dot{x}=h(x)-\sum_{i=1}^{n} u_{i}
$$


Let us assume that the instantaneous utility that agent $i$ derives from the consumption of the stock is given by

$$
L_{i}\left(u_{1}, \cdots, u_{n}\right)
$$

so that his utility functional becomes

$$
\mathcal{J}_{i}=\int_{0}^{\infty} L_{i}\left(u_{1}, \cdots, u_{n}\right) \mathrm{e}^{-\rho t} \mathrm{~d} t .
$$

This functional covers several cases. The fish catch can be sold in an imperfect output market. In that case the price of the fish depends on the total quantity produced by all fisheries and therefore the function $L_{i}(u)$ depends on the extraction rates of all players. In case of a duopoly market with linear demand this problem was studied in [1].

Alternatively, the function $L_{i}(u)$ can only depend on the exploitation rate of player $i$. In this case each agents maximizes the present value of future utility derived from consuming the fish. This formulation was dealt with in [8].

3.4.1 Duopoly. Let us start with the duopoly model studied by [1]. The number of players is assumed to be two, $n=2$, and the utility (revenue) functions are given by

$$
L_{i}\left(u_{i}, u_{j}\right)=\left(a-b\left(u_{i}+u_{j}\right)\right) u_{i}
$$

Moreover, the production function is specified as

$$
h(x)= \begin{cases}\delta x & \text { for } x \leq \frac{x_{\max }}{2} \\ \delta\left(x_{\max }-x\right) & \text { for } x>\frac{x_{\max }}{2}\end{cases}
$$

It is assumed that marginal product is large enough to satisfy $\delta>\max \left\{\frac{5 \rho}{2}, \frac{10 a}{9 b}\right\}$. Hence, the production function is piecewise linear and the utility function is quadratic. The function $\mathcal{P}_{i}$ is given by

$$
\mathcal{P}_{i}\left(x, p_{i}, u_{i}\right)=-a u_{i}+b\left(u_{i}^{2}+u_{i} u_{j}\right)+p_{i}\left(h(x)-u_{i}-u_{j}\right) .
$$

This yields that $p_{i}=q_{i}\left(x, u_{i}, u_{j}\right)=-a+2 b u_{i}+b u_{j}(x), u_{i}=v_{i}\left(x, u_{j}, p_{i}\right)=\left(p_{i}+a\right) /(2 b)-(1 / 2) u_{j}$ Applying equation (5) and assuming symmetry we obtain

$$
\frac{\mathrm{d} u}{\mathrm{~d} x}=\frac{\left(\frac{a}{3 b}-u\right)\left(h^{\prime}(x)-\rho\right)}{h(x)-\frac{8}{3} u+\frac{a}{3 b}} .
$$

The corresponding auxiliary systems becomes

$$
\left\{\begin{array}{l}
x^{\prime}=h(x)-\frac{8}{3} u+\frac{a}{3 b}, \\
u^{\prime}=\left(\frac{a}{3 b}-u\right)\left(h^{\prime}(x)-\rho\right) .
\end{array}\right.
$$

Given the assumption on the production function, marginal product is piecewise constant, i.e. $h^{\prime}(x)=\delta$ or $h^{\prime}(x)=-\delta$. From the auxiliary system we immediately find that $\left(u_{0}=\frac{a}{3 b}, x_{0}=\frac{5}{9} \frac{a}{b \delta}\right)$ and $\left(u_{0}=\right.$ $\left.\frac{a}{3 b}, x_{0}=\frac{9 b \delta-5 a}{9 b \delta}\right)$ are steady states.

Since the duopoly game is of the linear quadratic type, it makes sense to look for a linear Markov equilibrium. The linear equilibrium, however, cannot be applied for the entire state space. Whenever the stock 


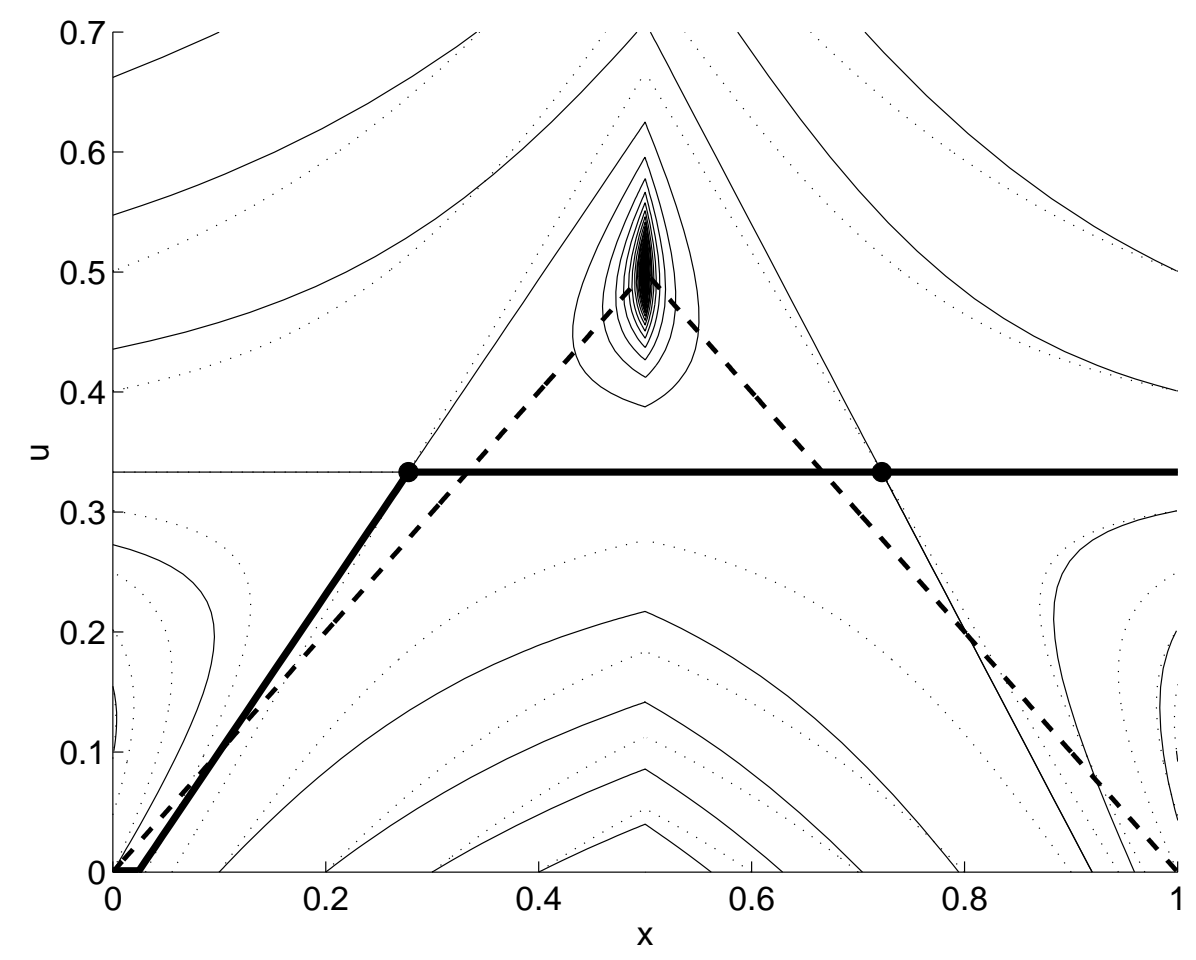

Figure 3: Solutions of the auxiliary system (solid) and level curves of the value function (dotted) in the symmetric two player duopoly with production function $h(x)=\delta \min \{x, 1-x\}$. Parameters are $a=b=$, $\delta=2, \rho=1 / 2$. The highlighted curve is the piecewise linear solution described in the text.

level is large enough it is optimal for the firms to chose the steady state level $u_{0}=\frac{a}{3 b}$ and stay at this level. Prior to reaching this upper steady state firms can choose linear Markov strategies. They can be derived making use of (13). Let us assume that in the appropriate state space range strategies are linear, and that there $h(x)=\delta x$. This implies that $\frac{\mathrm{d} u}{\mathrm{~d} x}=\alpha$ where $\alpha$ is a constant. Using the relationship

$$
\frac{\mathrm{d} u}{\mathrm{~d} x}=\frac{\left(\frac{a}{3 b}-u(x)\right)(\delta-\rho)}{\delta x-\frac{8}{3} u(x)+\frac{a}{3 b}}=\alpha,
$$

we find that $\alpha=\frac{3}{4}\left(\delta-\frac{\rho}{2}\right)$ and that the linear strategies are given by

$$
u(x)=\frac{3}{4}\left(\delta-\frac{\rho}{2}\right) x-\frac{a}{12 b \delta}\left(\delta-\frac{5 \rho}{2}\right) .
$$

It is now easily seen that the equilibrium strategy consists of three parts. For stock levels below $x_{1}=$ $\frac{a}{9 b \delta} \frac{\delta-5 \rho / 2}{\delta-\rho / 2}$, equilibrium production is zero. For levels above $x_{2}=\frac{5}{9} \frac{a}{b \delta}$, the optimal policy of the firms is to choose $u_{0}=\frac{a}{3 b}$. For intermediate levels it is optimal to choose the linear Markov strategy given by (15). This equilibrium is illustrated in figure 3 . Note that the condition $\delta>\frac{10 a}{9 b}$ ensures that $x_{2}<1 / 2$. 
3.4.2 Constant relative risk aversion. We now proceed with the case in which each agent has a constant relative risk aversion utility function. Let $0<\sigma<1$ and specify the utility functional of player $i$ as

$$
\partial_{i}=\int_{0}^{\infty} \frac{u_{i}^{1-\sigma}}{1-\sigma} \mathrm{e}^{-\rho t} \mathrm{~d} t
$$

The function $\mathcal{P}_{i}$ becomes

$$
\mathcal{P}_{i}=-\frac{u_{i}^{1-\sigma}}{1-\sigma}+p_{i}\left(h(x)-\sum_{j=1}^{n} u_{j}\right)
$$

From $\partial \mathcal{P}_{i} / \partial u_{i}$ we obtain $p_{i}=-u_{i}^{-\sigma}$ and $u_{i}=\left(-p_{i}\right)^{-1 / \sigma}$, and equation (5) yields

$$
\frac{\mathrm{d} u_{i}}{\mathrm{~d} x}\left(h(x)-\sum_{j=1}^{n} u_{j}\right)+\frac{u_{i}}{\sigma} \sum_{j \neq i} \frac{\mathrm{d} u_{j}}{\mathrm{~d} x}=\frac{u_{i}}{\sigma}\left(h^{\prime}(x)-\rho\right) .
$$

Symmetric strategies. If all players play the same strategy $u_{i}=u$, then equation (16) reduces to

$$
\frac{\mathrm{d} u}{\mathrm{~d} x}=\frac{u}{\sigma} \frac{h^{\prime}(x)-\rho}{h(x)+\left(\frac{n-1}{\sigma}-n\right) u} .
$$

The corresponding auxiliary system reads as

$$
\left\{\begin{array}{l}
x^{\prime}=\sigma h(x)+((n-1)-n \sigma) u \\
u^{\prime}=u\left(h^{\prime}(x)-\rho\right) .
\end{array}\right.
$$

The case $\sigma=(1-1 / n)$ is special, since then the system can be integrated analytically, yielding

$$
u(x)=C h(x)^{\frac{n}{n-1}} \exp \left(-\frac{n \rho}{n-1} \int_{x_{0}}^{x} h(t)^{-1} \mathrm{~d} t\right) .
$$

Compare equation (4) of [8].

Stability of steady states. For a symmetric Nash equilibrium strategy $u(x)$, it follows from equation (12) that the point $x=x_{0}$ is a steady state if

$$
u\left(x_{0}\right)=\frac{1}{n} h\left(x_{0}\right) .
$$

Introducing $\xi=x-x_{0}$ yields for the local dynamics at the steady state

$$
\frac{\mathrm{d}}{\mathrm{d} t} \xi=\left(h^{\prime}\left(x_{0}\right)-n u^{\prime}\left(x_{0}\right)\right) \xi+\mathrm{O}\left(\xi^{2}\right) .
$$

From equations (19) and (17), we find that

$$
u^{\prime}\left(x_{0}\right)=h^{\prime}\left(x_{0}\right)-\rho
$$

substitution yields

$$
\frac{\mathrm{d}}{\mathrm{d} t} \xi=\frac{1}{n-1}\left(n \rho-h^{\prime}\left(x_{0}\right)\right) \xi+\mathrm{O}\left(\xi^{2}\right) .
$$



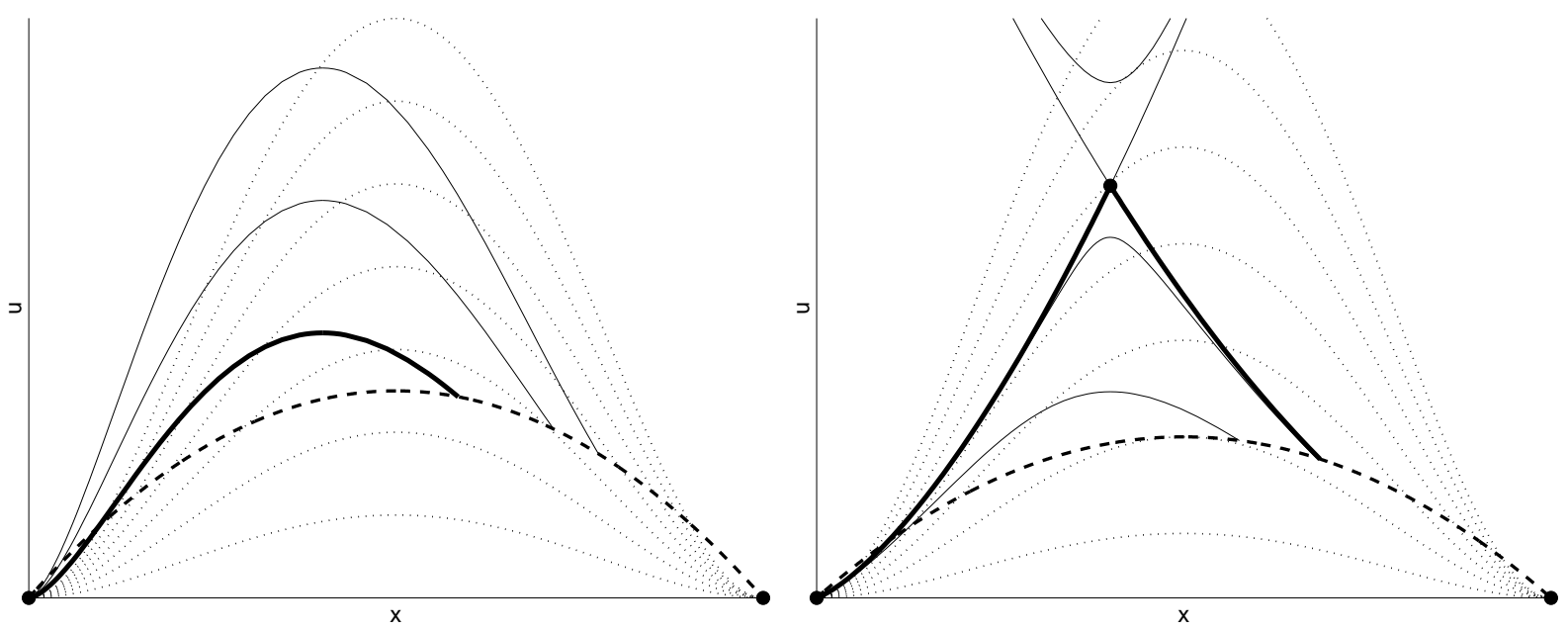

Figure 4: Solutions of the auxiliary system (solid) and level curves of the value function (dotted) in the symmetric two player case of the fishery model with production function $h(x)=x(1-x)$.

We find the condition that the steady state $x=x_{0}$ satisfying (19) is locally asymptotically stable if

$$
\rho<\frac{1}{n} h^{\prime}\left(x_{0}\right)
$$

and unstable if the inequality sign is reversed. In particular, if $h^{\prime}\left(x_{0}\right)<0$, then $\left(x_{0}, u\left(x_{0}\right)\right)$ is always an unstable equilibrium for the state dynamics. Moreover, the derivative $h^{\prime}(x)$ is bounded from above, and if $\rho>1 / n \cdot \max h^{\prime}(x)$, then the state dynamics do not have stable equilibria in the interior of the state space.

Case study. In the following, we shall restrict our attention to the analysis of the special case with $h(x)=$ $x(1-x)$ whenever illustrations are called for.

Local analysis of the auxiliary system. In the following, the auxiliary system is used to characterize the functions $u(x)$ for the case $h(x)=x(1-x)$.

The set $\mathcal{E}$ of the equilibria of the auxiliary system is

$$
\mathcal{E}=\left\{(0,0),(1,0),\left(x_{\rho}, \frac{\sigma}{n(1-\sigma)-1} h\left(x_{\rho}\right)\right)\right\}
$$

where $x_{\rho}=(1-\rho) / 2$ is the solution of $h^{\prime}(x)-\rho=0$. Note that the third equilibrium is in the quadrant $x>$ $0, u>0$ only if $\rho<1$ and $n>1 /(1-\sigma)$.

LEMMA 1. If $(x, u(x))$ is a solution of (17) that satisfies $0<u(x)<(1 / n) h(x)$, then the solution $(x(t), u(x(t))$ of the control problem of each player violates the transversality condition.

Proof

For an equilibrium $\left(x_{0}, u_{0}\right) \in \mathcal{E}$, introduce $\zeta=(\xi, \eta)=\left(x-x_{0}, u-u_{0}\right)$. The linearized system at $\left(x_{0}, u_{0}\right)$ then becomes

$$
\zeta^{\prime}=A \zeta=\left(\begin{array}{cc}
\sigma h^{\prime}\left(x_{0}\right) & n(1-\sigma)-1 \\
u_{0} h^{\prime \prime}\left(x_{0}\right) & h^{\prime}\left(x_{0}\right)-\rho
\end{array}\right) \zeta+\mathrm{O}\left(|\zeta|^{2}\right)
$$


Note that if $x_{0}=1$, the matrix $A$ takes the form

$$
A=\left(\begin{array}{cc}
-\sigma & n(1-\sigma)-1 \\
0 & -1-\rho
\end{array}\right)
$$

We have the following lemma:

LEMMA 2. The integral curves of the system $\zeta^{\prime}=\left(\begin{array}{cc}\alpha & \beta \\ 0 & \gamma\end{array}\right) \zeta$, with $\zeta=(\xi, \eta)$, satisfy the relation

$$
\xi=C|\eta|^{\frac{\alpha}{\gamma}}-\frac{\beta}{\gamma-\alpha} \eta
$$

where $C$ is an integration constant. This lemma yields that $\xi=C|\eta|^{\frac{\sigma}{1+\rho}}+\ldots$, and hence that the integral curve through $\left(\xi_{0}, \eta_{0}\right)$ is to lowest order given as

$$
\eta=\eta_{0}\left(\frac{\xi}{\xi_{0}}\right)^{\frac{1+\rho}{\sigma}}+\ldots
$$

The state dynamics at the equilibrium read as

$$
\frac{\mathrm{d}}{\mathrm{d} t} \xi=-\xi-n \eta_{0}\left(\frac{\xi}{\xi_{0}}\right)^{\frac{1+\rho}{\sigma}}+\ldots ;
$$

as $(1+\rho) / \sigma>1$, we have that $\xi(t)=\mathrm{e}^{-t} \xi_{0}+\ldots$, and consequently $u(t)=\eta(t)=\eta_{0} \mathrm{e}^{-\frac{1+\rho}{\sigma} t}$. Recalling that $p=-u^{-\sigma}$, we finally obtain

$$
p(t)=p_{0} \mathrm{e}^{(1+\rho) t} .
$$

Hence for all solutions of the auxiliary system that tend to the equilibrium $\left(x_{0}, u_{0}\right)=(1,0)$, the transversality condition $\lim _{t \rightarrow \infty} p(t) \mathrm{e}^{-\rho t}=0$ is violated.

Asymmetric strategies. Here the assumption is dropped that the players play symmetric strategies; for simplicity, we restrict to the two-player case $n=2$ and assume that $1-\sigma=1 / 2$ holds. Equation (16) gives rise to the system

$$
\begin{aligned}
& \left(h-u_{1}-u_{2}\right) \frac{\mathrm{d} u_{1}}{\mathrm{~d} x}+2 u_{1} \frac{\mathrm{d} u_{2}}{\mathrm{~d} x}=2\left(h^{\prime}-\rho\right) u_{1}, \\
& 2 u_{2} \frac{\mathrm{d} u_{1}}{\mathrm{~d} x}+\left(h-u_{1}-u_{2}\right) \frac{\mathrm{d} u_{2}}{\mathrm{~d} x}=2\left(h^{\prime}-\rho\right) u_{2} .
\end{aligned}
$$

It is convenient to consider instead of $u_{1}$ and $u_{2}$ the dependent quantities $v=u_{1}-u_{2}$ and $w=u_{1}+u_{2}$; for them, the equations take the simpler form

$$
\begin{aligned}
\frac{\mathrm{d} w}{\mathrm{~d} x} & =2 \frac{h^{\prime}-\rho}{\Delta}\left(h w-2 w^{2}+v^{2}\right), \\
\frac{\mathrm{d} v}{\mathrm{~d} x} & =2 \frac{h^{\prime}-\rho}{\Delta}(h-w) v,
\end{aligned}
$$

with $\Delta(x)=h^{2}-2 h w+v^{2}$. The auxiliary version of this system of equations is

$$
\begin{aligned}
x^{\prime} & =\Delta=h^{2}-2 h w+v^{2}, \\
w^{\prime} & =2\left(h^{\prime}-\rho\right)\left(h w-2 w^{2}+v^{2}\right), \\
v^{\prime} & =2\left(h^{\prime}-\rho\right)(h-w) v .
\end{aligned}
$$


Note that the plane $v=0$, corresponding to the symmetric case $u_{1}=u_{2}$, is invariant under the flow of the auxiliary system; in other words, that case is nested in the present one.

We will not give a full analysis of this system, leaving that to future work. However, we would like to point out one consequence of the equation $v^{\prime}=2\left(h^{\prime}-\rho\right)(h-w) v$. Recall that $x^{\prime}=h-w$; hence, if the system is on a time path for which the stock decreases, the factor $h-w<0$, and the sign of $v^{\prime} / v$ is the opposite of $h^{\prime}-\rho$.

In the example above, the factor $h^{\prime}-\rho$ is positive for small $\rho$ and small $x$, and it follows that then the differences between strategies decay exponentially if the stock decreases towards an equilibrium close to $x=0$. Conversely, if $\rho$ sufficiently large, differences between strategies increase exponentially, which can be seen as a mad scramble to exploit the last remnants of the stock.

\section{Conclusions}

In this article, a framework has been elaborated to find necessary conditions for Markov Nash equilibrium strategies in differential games with a single state variable. The Nash equilibria have been characterized as solutions of a system of explicit first order ordinary differential equations, usually nonlinear.

By analyzing a series of classical examples, we have shown that this characterization can be used to find both direct analytic information, by integration of the equations, and indirect qualitative information, by a geometric analysis of the solution curves of an auxiliary system in the phase space.

Additionally, we have addressed the issues of continuity and differentiability of Markov strategies in this class of differential games. Our simple approach is capable enough to deliver interesting insights into a large class of capital accumulation games. 


\section{References}

[1] H. Benchekroun, Unilateral production restrictions in a dynamic duopoly, Journal of Economic Theory 111 (2003), 214-239.

[2] J. Benhabib and R. Radner, The joint exploitation of a productive asset: a game theoretic approach, Economic Theory 2 (1992) 155-190.

[3] W.A. Brock and A.G. Malliaris, Differential equations, stability and chaos in dynamical systems, NorthHolland, 1989.

[4] W.A. Brock and D. Starrett, Nonconvexities in ecological management problems, University of Wisconsin, http://www.ssc.wisc.edu/econ/archive, 1999.

[5] S. Clemhout and H.Y. Wan, Differential Games - Economic Applications, Handbook of Game Theory (R.J. Aumann and S. Hart, eds.), North-Holland, 1994, 801-825.

[6] W.D. Dechert and W.A. Brock, The lake game, Preprint, 2000.

[7] E.J. Dockner, N.V. Long and G. Sorger, Analysis of Nash equilibria in a class of capital accumulation games, Journal of Economic Dynamics and Control 20 (1996), 1209-1235.

[8] E.J. Dockner and G. Sorger, Existence and properties of equilibria for a dynamic game on productivity assets, Journal of Economic Theory 71 (1996), 209-227.

[9] E.J. Dockner, N.V. Long, S. Jorgensen, and G. Sorger, Differential Games in Economics and Management Science, Cambridge University Press, 2000.

[10] E.J. Dockner and N.V. Long, International pollution control: cooperative versus non-cooperative strategies, Journal of Environmental Economics and Management 24 (1994), 13-29.

[11] E.J. Dockner and K. Nishimura, Private Investment of a Public Project? A Multiperiod Analysis, Working Paper, 2005.

[12] P.K. Dutta and R.K. Sundaram, How different can strategic models be?, Journal of Economic Theory 60 (1993), 413-426.

[13] M. Eswaran and T. Lewis, Appropriability and the extraction of a common property resource, Economica 51 (1984), 393-400.

[14] C. Fershtman and S. Nitzan, Dynamic voluntary provision of public goods, European Economic Review 35 (1991), 1057-1067.

[15] K.-G. Mäler, A. Xepapadeas, and A. de Zeeuw, The economics of shallow lakes, Environmental \& Resource Economics 9 (2003), 603-624.

[16] D. Levhari and L. Mirman, The great fish war: an example using a dynamic Cournot Nash solution, Bell Journal of Economics 11 (1980), 322-334.

[17] G. Kossioris, M. Plexousakis, A. Xepapadeas, A. de Zeeuw and K.-G. Mäler, Feedback Nash equilibria for non-linear differential games in pollution control, Preprint, (2006). 
[18] L.M. Marx and S.A. Matthews, Dynamic Voluntary Contribution to a Public Project, Review of Economic Studies 67 (2000), 327-358.

[19] J.P. Rincon-Zapatero, J. Martinez and G. Martin-Herran, New method to characterize subgame perfect Nash equilibria in differential games, Journal of Optimization Theory and Applications 96 (1998), $377-$ 395.

[20] R.K. Sundaram, Perfect equilibria in non-randomized strategies in a class of symmetric games, Journal of Economic Theory 47 (1989), 153-177.

[21] F.O.O. Wagener, Skiba points and heteroclinic bifurcations, with applications to the shallow lake system, Journal of Economic Dynamics and Control 27 (2003), 1533-1561.

[22] F. Wirl, Dynamic voluntary provision of public goods: Extension to nonlinear strategies, European Journal of Political Economy 12 (1996), 555-560.

[23] A. Xepapadeas, A. de Zeeuw, and K.-G. Mäler, Feedback equilibria for a class of non-linear differential games in resource economics, The Beijer Institute of Ecological Economics, Discussion paper 152 (2002). 


\section{Appendix}

Theorem 3 uses the assumption that the value function for each player is continuous. Here we discuss regularity conditions for the value function to be continuous within the framework of an optimal control problem. The agent's control problem consists of maximizing

$$
\mathcal{J}[x, u]=\int_{0}^{\infty} g(x, u) \mathrm{e}^{-\rho t} \mathrm{~d} t
$$

under the constraint that

$$
\dot{x}=f(x, u) .
$$

Here $x:[0, \infty) \rightarrow \mathcal{X}$ and $u:[0, \infty) \rightarrow \mathcal{U}$, with $\mathcal{X}$ an open subset of $\mathbb{R}^{m}$ and $\mathcal{U}$ the closure of an open subset of $\mathbb{R}^{k}$. We shall denote by $\mathcal{D}_{\xi}$ the set of all such functions $x$ and $u$ that satisfy equation (21) almost everywhere such that $u$ is integrable, $x$ absolutely continuous and $x(0)=\xi$.

Recall that the value function can be defined as

$$
V(\xi)=\sup _{\mathcal{D}_{\xi}} \mathcal{J}[x, u] .
$$

Loosely speaking, a system is locally controllable if any point in the neighborhood of a given point can be reached by choosing the control function $u$ appropriately. The following definition makes this notion precise.

DEFINITION. (Bounded controllability at a point) We shall call the system $\dot{x}=f(x, u)$ boundedly controllable at $x_{0}$, if for every $\eta>0$ there is a $\sigma_{0}=\sigma_{0}(\eta)>0$ such that for every $0<\sigma \leq \sigma_{0}$, there an open neighborhood $U \subset X$ of $x_{0}$, depending on $\eta$ and $\sigma$, such that the following holds.

If $t_{1}, t_{2}$ and $x_{1}, x_{2} \in U$ are such that $\left|t_{1}-t_{2}\right|<\sigma$, there is an integrable function $u:\left(t_{1}, t_{2}\right) \rightarrow \mathcal{U}$ such that $|u(t)|<\eta$, and an absolutely continuous function $x:\left(t_{1}, t_{2}\right) \rightarrow U$, that satisfy $x\left(t_{1}\right)=x_{1}, x\left(t_{2}\right)=x_{2}$ and

$$
\dot{x}=f(x, u),
$$

almost everywhere on $\left(t_{1}, t_{2}\right)$.

With this definition in place, the criterion that ensures continuity of the value function takes now a rather simple form.

THEOREM 4. If the system $\dot{x}=f(x, u)$ is boundedly controllable at $\xi$, then the value function $V$ is continuous at $\xi$.

\section{Proof}

Take $\varepsilon>0$ arbitrarily. We show that there is a neighborhood $U$ of $\xi$ such that if $\tilde{\xi} \in U$, then $V(\xi)-\varepsilon<$ $V(\tilde{\xi})<V(\xi)+\varepsilon$.

By the definition of value function, we can find $\left(x_{1}, u_{1}\right) \in \mathcal{D}_{\xi}$, such that

$$
\mathcal{\partial}\left[x_{1}, u_{1}\right]>V(\xi)-\frac{\varepsilon}{3} .
$$


Take $\eta>0, \delta>0$ and let $B_{\delta}(\xi)=\{x:|x-\xi|<\delta\}$. Introduce

$$
M=\sup _{|x-\xi|<\delta,|u|<\eta}|g(x, u)|,
$$

and

$$
\sigma_{1}=\frac{\varepsilon}{3} \min \left\{\frac{1}{M}, \frac{1}{\rho V(\xi)}\right\}
$$

Choose $\sigma>0$ such that $\sigma \leq \min \left\{\sigma_{0}(\eta), \sigma_{1}\right\}$.

Find an open neighborhood $U \subset B_{\delta}$ of $\xi$ such that for every $\tilde{\xi} \in U$, there is an integrable function $\tilde{u}$, bounded by $\eta$, and an absolutely continuous function $\tilde{x}$, both defined on $(0, \sigma)$ such that $\dot{\tilde{x}}=f(\tilde{x}, \tilde{u})$ almost everywhere and $\tilde{x}(0)=\tilde{\xi}, \tilde{x}(\sigma)=\xi$.

We construct $\left(x_{2}, u_{2}\right) \in \mathcal{D}_{\tilde{\xi}}$ as follows:

$$
x_{2}(t)=\left\{\begin{array}{ll}
\tilde{x}(t) & 0 \leq t<\sigma, \\
x_{1}(t-\sigma), & t \geq \sigma .
\end{array} \quad u_{2}(t)= \begin{cases}\tilde{u}(t), & 0 \leq t<\sigma, \\
u_{1}(t-\sigma), & t \geq \sigma .\end{cases}\right.
$$

It then follows that

$$
V(\tilde{\xi}) \geq \mathcal{J}\left[x_{2}, u_{2}\right]=\int_{0}^{\sigma} g(\tilde{x}, \tilde{u}) \mathrm{e}^{-\rho t} \mathrm{~d} t+\int_{\sigma}^{\infty} g\left(x_{1}(t-\sigma), u_{1}(t-\sigma)\right) \mathrm{e}^{-\rho t} \mathrm{~d} t .
$$

The summands can be estimated as

$$
\int_{0}^{\sigma} g(\tilde{x}, \tilde{u}) \mathrm{e}^{-\rho t} \mathrm{~d} t \geq-M \sigma \geq-\frac{\varepsilon}{3}
$$

and

$$
\begin{aligned}
\int_{\sigma}^{\infty} g( & \left.x_{1}(t-\sigma), u_{1}(t-\sigma)\right) \mathrm{e}^{-\rho t} \mathrm{~d} t \\
& =\mathrm{e}^{-\rho \sigma} \mathfrak{J}\left[x_{1}, u_{1}\right]>\mathrm{e}^{-\rho \sigma}\left(V(\xi)-\frac{\varepsilon}{3}\right) \\
& =V(\xi)-\frac{\varepsilon}{3}-\left(1-\mathrm{e}^{-\rho \sigma}\right) V(\xi) \geq V(\xi)-\frac{\varepsilon}{3}-\rho \sigma V(\xi) \\
& \geq V(\xi)-\frac{2 \varepsilon}{3} .
\end{aligned}
$$

Adding these, we arrive at

$$
V(\tilde{\xi})>V(\xi)-\varepsilon
$$

Completely analogously, we show that $V(\xi)>V(\tilde{\xi})-\varepsilon$. Combining these inequalities yields that

$$
-\varepsilon<V(\tilde{\xi})-V(\xi)<\varepsilon .
$$

This shows that $V$ is continuous at $\xi$. 